\title{
Spin Currents and Spin Dynamics in Time-Dependent Density-Functional Theory
}

\author{
K. Capelle \\ Departamento de Química e Física Molecular, Instituto de Química de São Carlos, Universidade de São Paulo, \\ Caixa Postal 780, São Carlos SP, 13560-970 Brazil \\ G. Vignale \\ Department of Physics and Astronomy, University of Missouri-Columbia, Columbia, Missouri 65211 \\ B. L. Györffy \\ H. H. Wills Physics Laboratory, University of Bristol, Bristol, BS8 1TL, United Kingdom
}

(Received 21 May 2001; published 26 October 2001)

\begin{abstract}
We derive and analyze the equation of motion for the spin degrees of freedom within time-dependent spin-density-functional theory (TD-SDFT). The results are (i) a prescription for obtaining many-body corrections to the single-particle spin currents from the Kohn-Sham equation of TD-SDFT, (ii) the existence of an exchange-correlation (xc) torque within TD-SDFT, (iii) a prescription for calculating, from TD-SDFT, the torque exerted by spin currents on the spin magnetization, (iv) a novel exact constraint on approximate xc functionals, and (v) the discovery of serious deficiencies of popular approximations to TD-SDFT when applied to spin dynamics.
\end{abstract}

DOI: 10.1103/PhysRevLett.87.206403

PACS numbers: 71.15.Mb, 72.25.-b, 73.40.-c, 75.40.Gb

The dynamics of the spin degrees of freedom is responsible for such diverse phenomena as spin wave excitations, Bloch wall motion, spin-polarized currents, spin injection, and spin filtering; concepts and phenomena which are important, e.g., in the growing field of spintronics [1]. The calculation of spin dynamics within density-functional theory (DFT) has consequently received much attention [2]. The most popular DFT method for a first-principles treatment of the spin degrees of freedom is spin-densityfunctional theory (SDFT, see Refs. [3,4] for reviews.) SDFT has led to versatile and powerful schemes for the calculation of, e.g., total energies, spin densities, and spin-resolved single-particle band structures, but its traditional (i.e., ground state) formulation is applicable only to static situations. This situation has changed with the advent of time-dependent DFT (TD-DFT) [5], which has brought dynamical phenomena within reach of DFT.

In this Letter, we first derive the equation of motion for the spin magnetization from TD-SDFT, including exchange-correlation (xc) effects. We then show how this equation can be used to obtain information on the manybody spin current. Although one might think that a calculation of spin currents would require the more complex formalism of time-dependent current-density-functional theory (CDFT), we find that this is not entirely true: as a consequence of the continuity equation TD-SDFT suffices to calculate the spin currents in several cases of great practical interest.

Numerical applications of TD-SDFT, as of any other DFT, require knowledge of the xc potentials, which contain all many-body effects beyond the Hartree approximation. In traditional DFT many exact properties of these xc potentials are known, and greatly aid the construction of good approximations $[3,4]$, but the same is not true in the time-dependent case, where properties of the xc potentials are just beginning to be explored [6]. As a by-product, our analysis reveals a previously unknown exact property of the xc potentials of TD-SDFT, which strongly constrains suitable approximations.

In TD-SDFT the fundamental variables are the timedependent particle density,

$$
n(\mathbf{r}, t)=\sum_{i}^{N}\left\langle\Psi\left|\delta\left(\mathbf{r}-\mathbf{r}_{i}\right)\right| \Psi\right\rangle
$$

and the time-dependent magnetization (or spin) density

$$
\mathbf{m}(\mathbf{r}, t)=\mu_{0} \sum_{i}^{N}\left\langle\Psi\left|\hat{\boldsymbol{\sigma}}_{i} \delta\left(\mathbf{r}-\mathbf{r}_{i}\right)\right| \Psi\right\rangle,
$$

where $\Psi=\Psi\left(\mathbf{r}_{1}, \ldots, \mathbf{r}_{N}, t\right)$ is the many-body wave function (spin coordinates are suppressed for brevity), $\mu_{0}=$ $q \hbar /(2 m c)$ is the Bohr magneton, $\hat{\boldsymbol{\sigma}}_{i}$ is the vector of Pauli matrices, and $N$ is the particle number. Here and below all operators are taken to be independent of time, i.e., the time dependence of expectation values results exclusively from that of the wave function. In TD-SDFT these expectation values are not calculated with the many-body wave function $\Psi$, but from the solutions of a noninteracting Hamiltonian, containing suitably chosen effective electrostatic and magnetic fields $v_{s}(\mathbf{r}, t)$ and $\mathbf{B}_{s}(\mathbf{r}, t)$,

$$
\hat{H}^{\mathrm{KS}}=\sum_{i}^{N}\left[-\frac{\hbar^{2} \nabla_{i}^{2}}{2 m}+v_{s}\left(\mathbf{r}_{i}, t\right)-\mu_{0} \hat{\sigma}_{i} \cdot \mathbf{B}_{s}\left(\mathbf{r}_{i}, t\right)\right] .
$$

Equation (3) defines the Kohn-Sham (KS) Hamiltonian of TD-SDFT. The solution of $\left[i \hbar \partial /(\partial t)-\hat{H}^{\mathrm{KS}}\right] \Phi=0$, i.e., the Slater determinant $\Phi\left(\mathbf{r}_{1}, \ldots, \mathbf{r}_{N}, t\right)$, reproduces the correct particle and spin densities as functions of both time and position. By contrast, the current 


$$
\hat{\mathbf{j}}(\mathbf{r})=\frac{\hbar}{2 m i} \sum_{i}^{N} \nabla_{i} \delta\left(\mathbf{r}-\mathbf{r}_{i}\right)+\delta\left(\mathbf{r}-\mathbf{r}_{i}\right) \nabla_{i}
$$

is not among the basic variables of TD-SDFT, so that $\mathbf{j}(\mathbf{r}, t)=\langle\Psi|\hat{\mathbf{j}}(\mathbf{r})| \Psi\rangle \neq\langle\Phi|\hat{\mathbf{j}}(\mathbf{r})| \Phi\rangle=\mathbf{j}^{\mathrm{KS}}(\mathbf{r}, t)$.

The equations of motion for the fundamental density variables describing the electronic degrees of freedom, $n(\mathbf{r}, t)$ and $\mathbf{m}(\mathbf{r}, t)$, can be calculated directly from the commutator with the Kohn-Sham Hamiltonian. For the time evolution of $\mathbf{m}(\mathbf{r}, t)$, one finds

$$
\frac{d \mathbf{m}(\mathbf{r}, t)}{d t}+\hat{\nabla} \cdot \mathbf{J}^{\mathrm{KS}}(\mathbf{r}, t)=\frac{q}{2 m c} \mathbf{m}(\mathbf{r}, t) \times \mathbf{B}_{s}(\mathbf{r}, t) .
$$

The second term on the left-hand side, which stems from the commutator with the kinetic energy, describes spin currents arising because the electrons carry their spin with them as they move around. Here

$$
\mathbf{J}^{\mathrm{KS}}(\mathbf{r}, t):=\mu_{0} \sum_{i}^{N}\left\langle\Phi\left|\hat{\boldsymbol{\sigma}}_{i} \otimes \hat{\mathbf{j}}_{i}(\mathbf{r})\right| \Phi\right\rangle
$$

is the KS spin-current tensor, defined via the tensor product of the spin vector $\hat{\sigma}_{i}$ and the orbital current $\hat{\mathbf{j}}_{i}(\mathbf{r})$ of particle $i$ [7]. (We use a capital $\mathbf{J}$ to denote spincurrent tensors, and a lower case $\mathbf{j}$ for the corresponding orbital currents.) Equation (5) is a formally exact TD-SDFT representation of the time evolution of the spin degrees of freedom. Many-body effects enter Eq. (5) via the effective magnetic field $\mathbf{B}_{s}$, which is defined as $\mathbf{B}_{s}(\mathbf{r}, t)=\mathbf{B}(\mathbf{r}, t)+\mathbf{B}_{\mathrm{xc}}(\mathbf{r}, t)$, where $\mathbf{B}(\mathbf{r}, t)$ is the external magnetic field, and $\mathbf{B}_{\mathrm{xc}}(\mathbf{r}, t)$ is the exchangecorrelation magnetic field.

Of course, the equation of motion for $\mathbf{m}(\mathbf{r}, t)$ can also be derived from the many-body Hamiltonian. Since in the absence of relativistic effects the spin magnetization commutes with the particle-particle interaction, the result takes the same form as in the Kohn-Sham system,

$$
\frac{d \mathbf{m}(\mathbf{r}, t)}{d t}+\hat{\nabla} \cdot \mathbf{J}(\mathbf{r}, t)=\frac{q}{2 m c} \mathbf{m}(\mathbf{r}, t) \times \mathbf{B}(\mathbf{r}, t),
$$

up to the replacement of the effective by the external magnetic field, and the Kohn-Sham current $\mathbf{J}^{\mathrm{KS}}$ by the many-body current $\mathbf{J}$. Equation (7) is simply the continuity equation for the spin magnetization. Comparing Eqs. (5) and (7), one finds that

$$
\frac{q}{2 m c} \mathbf{m}(\mathbf{r}, t) \times \mathbf{B}_{\mathrm{xc}}(\mathbf{r}, t)=\hat{\nabla} \cdot\left[\mathbf{J}^{\mathrm{KS}}(\mathbf{r}, t)-\mathbf{J}(\mathbf{r}, t)\right]
$$

for all times $t$ and at every point $\mathbf{r}$. This equation is the central result of the present analysis. We now proceed to explore some of the consequences, practical and fundamental, of this result.

(i) The right-hand side of Eq. (8) contains the difference between the many-body spin current and its Kohn-Sham counterpart. This difference is, in the following, denoted $\mathbf{J}_{\mathrm{xc}}$, since it is the exchange-correlation contribution to the full current. The longitudinal part of $\mathbf{J}_{\mathrm{xc}}$, denoted $\mathbf{J}_{\mathrm{xc}}^{L}$, can be calculated directly from TD-SDFT by integrating Eq. (8). It is interesting to note that this many-body correction to the Kohn-Sham current follows from SDFT alone, without having to use CDFT.

$\mathbf{J}_{\mathrm{xc}}^{L}$ on its own is valuable information, but a full determination of the many-body current requires knowledge of the transverse correction also, and this can, at present, only be calculated within CDFT. For a large class of systems, however, $\mathbf{J}_{\mathrm{xc}}^{L}$ is the most relevant many-body correction. This includes all quasi-one-dimensional systems (e.g., conducting polymers), in which only the longitudinal component of the spin current is of interest, but also three-dimensional systems in which the current flows entirely in one direction, e.g., perpendicular to an interface. A particularly important example is provided by magnetoresistive devices in which the spin-current flow is perpendicular to the planes of a magnetic multilayer: such a current is purely longitudinal and can therefore be computed from Eq. (8).

(ii) The left-hand side of Eq. (8) is the torque locally exerted by the xc magnetic field on the spin configuration. The existence of this torque explains how TD-SDFT achieves a formally correct description of spin dynamics via Eq. (5) although the currents the spins are coupled to in that equation are the KS currents, and not the physical ones: the exchange-correlation torque (8) accounts for the difference between the many-body and the KS currents in Eq. (5). This torque arises from the component of $\mathbf{B}_{\mathrm{xc}}$ perpendicular to $\mathbf{m}(\mathbf{r}, t)$,

$$
\left|\mathbf{B}_{\mathrm{xc}}^{\perp}(\mathbf{r}, t)\right|=\frac{2 m c}{|q|} \frac{\left|\hat{\nabla} \cdot\left[\mathbf{J}^{\mathrm{KS}}(\mathbf{r}, t)-\mathbf{J}(\mathbf{r}, t)\right]\right|}{|\mathbf{m}(\mathbf{r}, t)|} .
$$

(iii) Equation (7) shows that for a spontaneously magnetic system [with $\mathbf{B}(\mathbf{r}, t)=0$ ] the quantity $-\hat{\nabla} \cdot \mathbf{J}(\mathbf{r}, t)$ can be interpreted as the torque exerted by the current on the spin magnetization. There has recently been much interest in such torques in connection with experiments on current-driven magnetization reversals [8]. Our Eq. (8) provides a way to calculate these torques from the SDFT quantities $\mathbf{B}_{\mathrm{xc}}(\mathbf{r}, t), \mathbf{m}(\mathbf{r}, t)$, and $\mathbf{J}^{\mathrm{KS}}(\mathbf{r}, t)$.

(iv) By integrating Eq. (8) over all space, one obtains

$$
\int d^{3} r \mathbf{m}(\mathbf{r}, t) \times \mathbf{B}_{\mathrm{xc}}(\mathbf{r}, t) \equiv 0,
$$

since the right-hand side vanishes by virtue of Gauss' theorem. Equation (10) has a simple physical interpretation: the self-consistent xc magnetic field cannot exert a net torque on the system as a whole. We refer to Eq. (10) in the following as the zero-torque theorem (ZTT). This terminology puts it on the same footing as the zero-force theorem $[5,6]$ which states that the electrostatic xc potential does not exert a net force on the system.

By integrating Eq. (8) over the volume of a finite system, one finds, instead of Eq. (10), 


$$
\frac{q}{2 m c} \int_{\Omega} d^{3} r \mathbf{m}(\mathbf{r}, t) \times \mathbf{B}_{\mathrm{xc}}(\mathbf{r}, t)=\int_{S} d \mathbf{S} \cdot \mathbf{J}_{\mathrm{xc}}(\mathbf{r}, t),
$$

where $\Omega$ is the volume of the system bounded by the surface $\mathbf{S}$. For a finite system the integral over $\mathbf{m} \times \mathbf{B}_{\mathrm{xc}}$ thus measures the net flux of xc spin current into and out of the system.

(v) Finally, through Eq. (10), Eq. (8) provides a stringent constraint on approximations for $\mathbf{B}_{\mathrm{xc}}$. Any such approximation which does not satisfy Eq. (10) is not consistent with the microscopic spin dynamics and the continuity equation for the spin magnetization. In the popular adiabatic local-density approximation (ALDA), for example, one takes as ones approximation the xc fields of static SDFT within the usual LDA, but evaluates these at the time-dependent densities [5]. Since in LDA $\mathbf{B}_{\mathrm{xc}}$ and $\mathbf{m}$ are by construction always parallel, one has, in the ALDA, $\mathbf{m}(\mathbf{r}, t) \times \mathbf{B}_{\mathrm{xc}}(\mathbf{r}, t) \equiv 0$ for all times $t$ and at any point $\mathbf{r}$, and Eq. (10) is trivially satisfied. The fact that the ALDA thus (fortuitously) satisfies the ZTT may explain the relative success LDA-based calculations have had in previous calculations of spin dynamics and other dynamical phenomena in TD-SDFT.

On the other hand, from Eq. (8), one finds that within the ALDA $\mathbf{J}_{\mathrm{xc}}^{L}(\mathbf{r}, t) \equiv 0$. Since one does not expect that many-body corrections to the longitudinal spin current vanish generally, their absence in the ALDA must be considered a defect of this approximation. Furthermore, from the equation of motion (5) it follows that in the ALDA the xc magnetic field does not make a contribution to spin dynamics, which is thus governed only by the noninteracting currents and the external magnetic field. In particular, for perfectly localized noninteracting moments (such that $\left.\mathbf{J}^{\mathrm{KS}} \equiv 0\right)$ and in the absence of an external magnetic field) $(\mathbf{B} \equiv 0)$, the ALDA yields $d \mathbf{m} / d t \equiv 0$, so that there is no spin dynamics at all. Within the linear-response approximation this problem of the ALDA has been noticed previously in Ref. [9]. The absence of spin dynamics for localized moments is another deficiency of the ALDA. This conclusion is consistent with the original phenomenological derivation of the noninteracting equation of motion [10], which shows that the spin dynamics of localized moments is driven by gradients of the magnetization, and these are not contained in the ALDA.

Typical LDA-based calculations of spin dynamics [2] avoid these problems by not proceeding entirely within the LDA (for example, LDA is combined with constraining fields which are not calculated self-consistently, or it is used only to determine parameters in a model Hamiltonian which itself is not of the LDA form). Any first-principles calculation fully within the LDA, however, is bound to run into the above problems.

We thus now turn to a discussion of popular improvements upon the LDA. We first note that any static functional that is invariant under the infinitesimal global spin rotation $\mathbf{m}(\mathbf{r}) \rightarrow \mathbf{m}(\mathbf{r})+\delta \boldsymbol{\varphi} \times \mathbf{m}(\mathbf{r})$ satisfies the ZTT, when used as input for an adiabatic approximation to TD-SDFT. An example is the exact-exchange (or optimized effective potential) method. Equation (10) was derived only for exchange and correlation together, i.e., it is not guaranteed a priori that an exchange-only approximation satisfies it. However, since the exact exchange term is rotationally invariant, satisfaction of the ZTT is automatic. No similar result holds for a general time-dependent, e.g., retarded, xc functional.

Next we consider gradient-dependent functionals. The static generalized gradient approximations of Ref. [11] depend only on $m_{z}$ and $\nabla m_{z}$. They also satisfy the ZTT identically, but at the price of again making $\mathbf{B}_{\mathrm{xc}} \| \mathbf{m}$. A gradient-dependent functional that depends on the full vector $\mathbf{m}$ and its derivatives was sketched in Ref. [12] and another will be constructed below. The general form of such functionals is

$$
E_{\mathrm{xc}}=\int d^{3} r e_{\mathrm{xc}}\left(n,|\mathbf{m}|, \nabla n, \nabla m_{x}, \nabla m_{y}, \nabla m_{z}\right),
$$

where the function $e_{\mathrm{xc}}$ need not be specified explicitly for the present purpose. By using the chain rule for functional derivatives, we write the derivative of the generic functional (12) as

$$
\begin{aligned}
-\mathbf{B}_{\mathrm{xc}}(\mathbf{r})= & \frac{\delta E_{\mathrm{xc}}}{\delta \mathbf{m}(\mathbf{r})}=\sum_{k} \mathbf{u}_{k} \frac{\delta}{\delta m_{k}(\mathbf{r})} \int d^{3} r^{\prime} e_{\mathrm{xc}}\left(\mathbf{r}^{\prime}\right) \\
& +\sum_{i k} \mathbf{u}_{k} \int d^{3} x \frac{\delta\left[\nabla m_{i}(\mathbf{x})\right]}{\delta m_{k}(\mathbf{r})} \\
& \times \frac{\delta}{\delta\left[\nabla m_{i}(\mathbf{x})\right]} \int d^{3} r^{\prime} e_{\mathrm{xc}}\left(\mathbf{r}^{\prime}\right)
\end{aligned}
$$

where the index $k$ labels components of the vector $\mathbf{m}$, and the unit vector in the direction of component $m_{k}$ is denoted $\mathbf{u}_{k}$. The derivative in the first term on the right-hand side acts only on $|\mathbf{m}|$, i.e., the terms $\nabla m_{k}$ are held fixed while differentiating. By performing the derivatives of $\nabla m_{i}$ with respect to $m_{k}$ and introducing the abbreviations $f(\mathbf{r})=\delta / \delta|\mathbf{m}(\mathbf{r})| \int d^{3} r^{\prime} e_{\mathrm{xc}}(\mathbf{r})$ and $\mathbf{g}_{k}(\mathbf{r})=$ $\delta / \delta\left[\nabla m_{k}(\mathbf{r})\right] \int d^{3} r^{\prime} e_{\mathrm{xc}}(\mathbf{r})$, one obtains

$$
-\mathbf{B}_{\mathrm{xc}}(\mathbf{r})=\mathbf{u}_{m} f(\mathbf{r})-\sum_{k} \mathbf{u}_{k} \nabla \cdot \mathbf{g}_{k}(\mathbf{r}),
$$

where $\mathbf{u}_{m}$ is the unit vector in the direction of $\mathbf{m}$. While the first term on the right-hand side is again parallel to $\mathbf{m}$, the second, arising from the gradients in Eq. (12), in general is not. Independently of the detailed form of the unspecified kernel $e_{\mathrm{xc}}$, we thus find that gradient-dependent functionals of all three components of $\mathbf{m}$ produce a $\mathbf{B}_{\mathrm{xc}}$ that is not necessarily parallel to $\mathbf{m}$. Such functionals thus have the potential to solve the problems encountered by the LDA: when used as input for an adiabatic approximation to TD-SDFT they can give rise to a nonzero $\mathbf{B}_{\mathrm{xc}}^{\perp}(\mathbf{r}, t)$ and hence, by virtue of Eqs. (5), (8), and (9), to nontrivial spin dynamics for localized moments, and to a nonzero $\mathbf{J}_{\mathrm{xc}}^{L}(\mathbf{r}, t)$. 
However, the ZTT constrains the dependence of the functional on the gradients of $m_{x}, m_{y}$, and $m_{z}$. In order to illustrate this with a simple example, relevant for the calculation of spin waves, we write $\mathbf{B}_{\mathrm{xc}}(\mathbf{r})=$ $\int d r^{\prime} \hat{K}[\mathbf{m}, n]\left(\mathbf{r}, \mathbf{r}^{\prime}\right) \mathbf{m}\left(\mathbf{r}^{\prime}\right)$, where the kernel $\hat{K}\left(\mathbf{r}, \mathbf{r}^{\prime}\right)$ is, for weakly inhomogeneous systems, a short-range function of $\left|\mathbf{r}-\mathbf{r}^{\prime}\right|$. We can then expand $\mathbf{m}\left(\mathbf{r}^{\prime}\right)$ to second order in $\left(\mathbf{r}-\mathbf{r}^{\prime}\right)$,

$$
\begin{aligned}
\mathbf{m}\left(\mathbf{r}^{\prime}\right)= & \mathbf{m}(\mathbf{r})+\left(\mathbf{r}-\mathbf{r}^{\prime}\right) \nabla \otimes \mathbf{m}(\mathbf{r}) \\
& +\left(\mathbf{r}-\mathbf{r}^{\prime}\right)^{2} \nabla^{2} \mathbf{m}(\mathbf{r}),
\end{aligned}
$$

and obtain

$$
\mathbf{B}_{\mathrm{xc}}(\mathbf{r})=\hat{K}_{2} \mathbf{m}(\mathbf{r})+\hat{K}_{4} \nabla^{2} \mathbf{m}(\mathbf{r}) .
$$

Here $\hat{K}_{2}$ and $\hat{K}_{4}$ are proportional to the second and fourth moments of the tensor $\hat{K}\left(\left|\mathbf{r}-\mathbf{r}^{\prime}\right|\right)$, respectively. The ZTT is satisfied when these moments, which in general are tensors, reduce to scalars. The first term in (16) then recovers the Stoner approximation to the LDA, while the second is of the form of the terms appearing in the phenomenological Lifshitz equation of motion for slowly varying moments [cf. Eq. (6.98) of Ref. [10] ]. Work to construct explicit expressions for these moments is in progress.

Up to this point we have considered conventional static SDFT only insofar as it provides the input for adiabatic approximations to TD-SDFT. We now take a brief look at static SDFT in its own right. The equation of motion for $\mathbf{m}$ in static SDFT is simply $d \mathbf{m}(\mathbf{r}) / d t=0$. Hence

$$
\hat{\nabla} \cdot \mathbf{J}^{\mathrm{KS}}(\mathbf{r})=\frac{q}{2 m c} \mathbf{m}(\mathbf{r}) \times \mathbf{B}_{s}(\mathbf{r}) .
$$

By comparing this with the corresponding many-body equation, which differs from it only through the replacement of $\mathbf{B}_{s}$ by the external field $\mathbf{B}$, and $\mathbf{J}^{\mathrm{KS}}$ by $\mathbf{J}$, one immediately finds

$$
\frac{q}{2 m c} \mathbf{m}(\mathbf{r}) \times \mathbf{B}_{\mathrm{xc}}(\mathbf{r})=\hat{\nabla} \cdot\left[\mathbf{J}^{\mathrm{KS}}(\mathbf{r})-\mathbf{J}(\mathbf{r})\right] .
$$

Since this is of the same structure as Eq. (8), our conclusions (i) to (v) hold in static SDFT too, however, now applied to equilibrium currents and magnetizations, and not to their dynamical counterparts. Perhaps the most interesting result for static SDFT is the existence of a component of $\mathbf{B}_{\mathrm{xc}}$ perpendicular to $\mathbf{m}$, since it implies that the prescription to take $\mathbf{B}_{\mathrm{xc}}$ locally parallel to $\mathbf{m}(\mathbf{r})$, which is employed in many recent calculations for noncollinear spin configurations [13], is consistent with Eq. (18) only to the extent that the right-hand side, i.e., the difference of the spin currents, can be neglected.

In summary, we have derived and analyzed the equation of motion for the spin degrees of freedom within
TD-SDFT. Our results have consequences for, e.g., the calculation of spin currents in polymers and multilayers, the use of the ALDA in investigations of spin dynamics, popular methods for treating noncollinear magnetism, and the construction of better density functionals.

K. C. thanks the Physics Departments of the University of Columbia, Missouri, USA, and of the University of Bristol, UK, for generous hospitality, the FAPESP for financial support, and L. N. Oliveira and J.C. Egues for useful discussions. This work resulted from a collaboration partially funded by the TMR network (Contract No. EMRX-CT96-0089). G. V. acknowledges support from NSF Grant No. DMR-0074959.

[1] G. A. Prinz Science, 282, 1660 (1998); R. Fiederling et al., Nature (London) 402, 787 (1999); J. C. Egues, Phys. Rev. Lett. 80, 4578 (1998); D. D. Awschalom and N. Samarth, J. Magn. Magn. Mater. 200, 130 (1999).

[2] G. M. Stocks et al., Philos. Mag. B, 78, 665 (1998); R. Gebauer and S. Baroni, Phys. Rev. B 61, 6459 (2000); V. P. Antropov et al., Phys. Rev. Lett. 75, 729 (1995); S. V. Halilov et al., Phys. Rev. B 58, 293 (1998); B. L. Györffy and J. B. Staunton, Phys. Rev. Lett. 69, 371 (1992); D. M. Edwards, J. Magn. Magn. Mater. 45, 151 (1984); Q. Niu and L. Kleinman, Phys. Rev. Lett. 80, 2205 (1998).

[3] R. M. Dreizler and E. K. U. Gross, Density Functional Theory (Springer-Verlag, Berlin, 1990).

[4] Density Functionals: Theory and Applications, edited by D. P. Joubert, Lecture Notes in Physics Vol. 500 (SpringerVerlag, Berlin, 1998).

[5] E. Runge and E. K. U. Gross, Phys. Rev. Lett. 52, 997 (1984); E. K. U. Gross, J. F. Dobson, and M. Petersilka, in Topics in Current Chemistry, edited by R. Nalewajski (Springer, Berlin, 1996), Vol. 181; K. Burke and E. K. U. Gross, in Density Functionals: Theory and Applications (Ref. [4]).

[6] P. Hessler, J. Park, and K. Burke, Phys. Rev. Lett. 82, 378 (1999); J. Dobson, Phys. Rev. Lett. 73, 2244 (1994); G. Vignale, Phys. Rev. Lett. 74, 3233 (1995).

[7] The tensor divergence, $\hat{\nabla} \cdot$, in Eq. (5) is defined as a contraction with respect to the second index, i.e., $\left(\hat{\nabla}_{r} \cdot \hat{T}\right)_{\alpha}:=\sum_{\beta} \partial /\left(\partial r_{\beta}\right) T_{\alpha \beta}(\mathbf{r})$, where $\hat{T}$ is an arbitrary tensor with components $T_{\alpha \beta}$.

[8] J. A. Katine et al., Phys. Rev. Lett. 84, 3149 (2000); J. C. Slonczewski, J. Magn. Magn. Mater. 159, L1 (1996).

[9] V. P. Antropov, B. N. Harmon, and A. N. Smirnov, J. Magn. Magn. Mater. 200, 148 (2000).

[10] E. M. Lifshitz and L.P. Pitaevskii, Statistical Physics Part II, Course of Theoretical Physics Vol. 9 (Reed Publishing, Oxford, 1980).

[11] J. P. Perdew, K. Burke, and M. Ernzerhof, Phys. Rev. Lett. 77, 3865 (1996); A. D. Becke, J. Chem. Phys. 107, 8554 (1997).

[12] K. Capelle and E. K. U. Gross, Phys. Rev. Lett. 78, 1872 (1997).

[13] L. M. Sandratskii, Adv. Phys. 47, 91 (1998); J. Sticht, K.-H. Höck, and J. Kübler, J. Phys. Condens. Matter 1, 8155 (1989). 